\title{
MEDIDAS HIGIENISTAS Y PLANES DE REFORMA URBANA EN EL TRÁNSITO DE LOS SIGLOS XIX AL XX EN LAS PRINCIPALES CIUDADES DE LA PROVINCIA DE ALICANTE
}

Gabino Ponce Herrero

Juan Manuel Dávila Linares

\section{RESUMEN}

En el tránsito del siglo XIX al XX, la carencia de viviendas y el hacinamiento son los rasgos más característicos de las sociedades urbanas analizadas, con un corolario de afecciones sanitarias. Tal circunstancia pudo ser paliada mediante planes de urbanismo fundamentados en aspectos higienistas. No obstante, las reformas fueron siempre a remolque de pequeños intereses económicos. La conciencia crítica de urbanistas y pensadores se enfrentó a la inercia de los políticos y a la incapacidad de algunas sociedades de vislumbrar la configuración de una ciudad moderna, a partir de las estructuras puede vislumbrar la configuración de una ciudad moderna, a partir de las estructuras pue-
blerinas en que vivían inmersas. Todo ello hizo que la respuesta a esas carencias acuciantes se viese siempre demorada o mal satisfecha.

Palabras clave: Reforma urbana e higienismo.

\section{ABSTRACT}

In the transition from the 19th to the 20th centuries the lack of dwellings and overcrowding are the most characteristic features of the urban societies analyzed, together with a corollary of health conditions. This problem was corrected urban plans based on aspects concerning hygiene aspects. However, the reforms lagged behind small economic interests. The critical awareness of urbanists and thinkers came up against the apathy of politicians as well as the fact that some societies were unable to discern the configuration of modern towns immersed, as they were, in village-like structures. Therefore, the solution to these acute shortenings always took a long time coming or when it did, was unsatisfactory.

Key words: Urban reform and hygiene aspects.

La progresiva concentración de población en las ciudades es un proceso que se generaliza en toda Europa en el tránsito de los siglos XIX al XX, sobre todo en los países indus- 
trializados, en los que el proceso muestra espectaculares índices de progresión. En los municipios de la provincia de Alicante tal fenómeno muestra una clara distinción entre aquéllos favorecidos por las rentas de situación de su emplazamiento y los ubicados en espacios marginales respecto a los ejes y focos de desarrollo.

En el primer caso destaca el conjunto de municipios ubicados en corredores bien comunicados, como el eje del Vinalopó, servido desde mediados del siglo pasado por el trazado del ferrocarril Alicante-Valencia-Madrid, beneficiados en su momento por la extraordinaria coyuntura de la expansión de la viticultura y la producción de vinos para la exportación; en general, el crecimiento demográfico fue tal que llegó a duplicar en algunas ciudades el número de habitantes y, con él, la extensión del callejero, apareciendo los primeros barrios modernos, sujetos a un planeamiento previo, en los que se deja notar la introducción de medidas de corte higienista que, aunque modestas, contrastan con las inexistentes o insuficientes en los callejeros tradicionales.

Experimentaron también destacados crecimientos las principales ciudades de la provincia, excepción hecha de Orihuela, que salda con un prolongado estancamiento poblacional todo el tránsito por la segunda mitad del siglo pasado. Frente a ésta, Alicante y Alcoy evidencian un notable crecimiento demográfico y urbano, debido en el primer caso al auge del comercio y, en el segundo caso, al despegue industrial desde mediados de la centuria. A ellas se suma Elche ya en el último tercio del siglo pasado, apoyada en la fabricación de calzado. En todos los casos, la bonanza económica generada supuso el arranque de un proceso de atracción de inmigrantes que contribuyó a reforzar el crecimiento vegetativo y, entre ambos, a proyectar el crecimiento urbano más allá de los límites tradicionales, en algún caso, como en Alicante, definido todavía por la muralla.

Evolución de la población en las principales ciudades de la provincia

\begin{tabular}{|c|c|c|c|c|}
\hline Año & Alicante & Elche & Alcoy & Orihuela \\
\hline 1835 & & & 20.924 & \\
1857 & 27.550 & 19.533 & 25.315 & 26.187 \\
1877 & 34.926 & 19.636 & 32.492 & 24.629 \\
1900 & 50.142 & 27.308 & 32.053 & 28.530 \\
1920 & 63.908 & 33.167 & 36.463 & 37.180 \\
\hline
\end{tabular}

Fuente: INE, censos, elab. Prop.

Ese proceso de concentración urbana y crecimiento selectivo de la población, general en las principales ciudades de España, llevó consigo la aparición de nuevos problemas derivados del fuerte incremento de las densidades humanas en las urbes. En Alcoy, en 1877 se registraban valores medios de 1.000 habitantes por Ha en los barrios obreros del Portal Nou, de la Sang o Cova Santa y calles adyacentes, rebasando incluso los 2.500 habitantes por Ha en barrios como el de Casas Nuevas o de Algezares ${ }^{1}$. A este respecto, resulta harto descriptiva la siguiente cita:

1 DÁVILA LINARES, J.M., Alcoy: desarrollo urbano y planeamiento, Alicante, Universidad de Alicante, 1993, 198 pp., p. 51. 
«En una calle que apenas mide cuatro metros y sobre un número muy limitado de palmos cuadrados, veíamos alzarse un edificio que, impulsado según parece por el orgullo o quien sabe si por la codicia de su amo, pretende llegar a los cielos como otra Babel, con olvido punible de todas las prescripciones higiénicas; (...)este edificio de atlética altitud y raquítica latitud presenta en su fachada un escaso número de aberturas (...). Si entramos allí, un hedor indefinible es lo que se percibe (...). Un visible número de puertas demuestran sin esfuerzo que sus habitantes no son pocos. La habitación del obrero tiene reducidísimo espacio (...); horroriza pensar cómo en una habitación donde apenas cabe poco más de una cama, de techo bajo y sin más abertura que la puerta de entrada, se entregan en brazos de Morfeo, cuatro seres (...). Tal es la habitación del obrero alcoyano, digo mal, el hormiguero donde, apilado, vive muriendo el que es la base del edificio social .... $\nu^{2}$

Así, la carencia de suelo urbano se erige en principal obstáculo para el normal desarrollo de las ciudades: en Alicante y otras ciudades españoles por hallarse todavía encorsetadas por la línea de muralla, y en Alcoy por la presencia de diversos barrancos, con profundos taludes, ciñendo el casco urbano. Menos determinantes, pero también condicionantes del desarrollo urbano, fueron en Elche el cauce del Vinalopó y los huertos de palmeras que rodean el sector oriental de la ciudad.

Consecuencia directa de ese hacinamiento fue la aparición de nuevos suburbios en la plena acepción de la palabra, esto es, barrios infradotados de todo tipo de servicios, con viviendas de escasas dimensiones apiñadas en edificios insalubres, con destacadas carencias sanitarias y de saneamiento. Por otro lado, se produce un crecimiento en altura de los edificios preexistentes del centro histórico, que duplican el número de plantas mediante añadidos de varias nuevas viviendas en las azoteas, en un proceso todavía visible por la yuxtaposición de diferentes estructuras y materiales. En otros casos, esos edificios antiguos son sustituidos por nuevas construcciones que, sobre la misma parcela de reducidas dimensiones y escaso frente de fachada, se levantan ganando altura, con viviendas alargadas hacia el interior de la parcela, escasamente ventiladas o beneficiadas por la luz del sol.

Aun así, el problema más acuciante es el de la escasez de viviendas y, sobre todo, de viviendas económicas. En las principales ciudades alicantinas se acometen desamortizaciones que afectan a propiedades de la Iglesia: el convento y huerto de los Franciscanos y el convento de los Dominicos en Alicante, el convento de San José y de la Merced en Elche y el convento de San Agustín en Alcoy (cuyo claustro acabó convertido en la Plaçeta de Dins), que permiten la liberación de pequeños lotes de suelo, ocupados de manera inmediata por nuevas calles y barriadas; se ha señalado este proceso como un pequeño ensanche interior, que permitió el crecimiento de las urbes sobre espacios intramuros. No obstante, esas reformas se mostraron insuficientes, tanto para aligerar las densidades humanas como para frenar los problemas higiénicos derivados de ella, que tuvieron su vertiente más trágica en la propagación de las epidemias de cólera de 1834, 1854, 1865 y de 1885, o con motivo de la fiebre amarilla de 1870, todas con gran incidencia urbana.

En esas circunstancias, las ciudades comienzan a dotarse a si mismas de instrumentos para paliar los problemas surgidos por el desbordamiento poblacional de los restringidos

2 AGUILAR MARTÍNEZ, F., Estado actual de las clases obreras de esta ciudad y medios que pudieran utilizarse para su perfeccionamiento en el orden físico y moral, Alcoy, Impr. La Revista de Alcoy, 1882, pp. 10-11. 
espacios urbanos, y para mitigar las secuelas de carácter sanitario inherentes al proceso. Desde el punto de vista edilicio se decide en muchos casos la demolición de la muralla (en Alicante en 1858), la acometida de reformas en el interior de las ciudades y la construcción de nuevos barrios planificados en los espacios periféricos, que recibieron la denominación genérica de Ensanches.

Ahora bien, tales procesos exigían, por un lado, un cuerpo legal suficiente para acometerlos y, por otro, el interés de la burguesía para invertir en ellos y beneficiarse de las plusvalías generadas por la apertura de nuevos espacios urbanos dentro y fuera de la ciudad tradicional. La evolución experimentada por los ensanches, evidenciada en el caso de Alicante, puso de manifiesto la insuficiencia de la legislación generada para resolverlos, al menos de manera efectiva conforme a sus planteamientos originales. También se observa, en los casos analizados, la incapacidad para llevar a efecto multitud de propuestas de corte higienista ligadas a actuaciones urbanas, en una época, por otro lado, fecunda en iniciativas y reflexiones urbanísticas, aunque trazadas siempre con una visión sectorial y sesgada del problema y, en su mayor parte, sin efectividad práctica. Será precisamente el desarrollo de la legislación sanitaria el detonante de la necesidad de redactar proyectos de saneamiento y reforma interior para buen número de ciudades españolas.

En las décadas centrales del siglo pasado se acometen en París las reformas de Haussmann, para modernizar una ciudad capital de un imperio, aquejada de los problemas ya expuestos de hacinamiento y carencias higiénicas: se pretendía recrear una escenografía acorde con la pujante burguesía y hacer más funcional la ciudad. No obstante, subyacían en esos proyectos otros objetivos más prosáicos, tales como poner en marcha un mecanismo legal que permitiera abordar los procesos de expropiación, de acuerdo con los «casatenientes», beneficiados por las plusvalías de la renta del suelo, derivadas de las obras de renovación de calles y avenidas. Por otro lado, prioritaria fue también la organización de un sistema policial y militar de represión, que exigía hacer accesible el centro histórico a las fuerzas de los cuerpos armados del Estado para sofocar movimientos sociales subersivos. En este sentido, ha de señalarse que, en 1868, triunfaba en Alicante La Gloriosa, revolución precedida de diversas revueltas sociales, o que en ese mismo año los republicanos se hacían con el poder en Alcoy y defendían la ciudad frente al acoso de las fuerzas gubernamentales. Así, en el paquete de intenciones de las reformas, en muchos casos amparadas en criterios higienistas, iban también claros intereses crematísticos y políticos.

\section{Las Topografías Médicas: antecedentes teóricos de los Proyectos de Saneamiento y} Reforma Interior

El deseo de mejorar las condiciones higiénicas y el grado de habitabilidad de los centros urbanos, favorece a principios de siglo la recuperación de ideas de saneamiento y reforma de aquellos sectores donde los problemas de deterioro, hacinamiento y pérdida de calidad de vida se hacían especialmente significativos ${ }^{3}$.

A finales del siglo XVIII cristaliza en España una línea de investigación desarrollada tiempo atrás en varios países europeos que se concreta en una corriente de pensamiento denominada higienismo. Esa corriente, desarrollada principalmente por médicos, tiene

3 En este sentido, es conveniente la lectura del completo estudio de LUIS URTEAGA, «Miseria, miasmas y microbios. Las topografías médicas y el estudio del medio ambiente en el siglo XIX, Revista Geocrítica, Univ. Barcelona, 1980, núm. 29, pp. 5-50. 
como principal objetivo la propuesta de medidas higiénico-sociales para la mejora de la salud y de las condiciones de existencia de la población, sobre todo en las ciudades industriales.

La existencia de relaciones entre la morbilidad, y por lo tanto la mortalidad, y el medio ambiente, es una idea que subyace en las teorías higienistas y que aparece generalmente aceptada por la mayoría de médicos desde finales del siglo XVIII. El estudio de estas relaciones se concretará durante el siglo XIX en la aparición de una serie de trabajos que tomarán el nombre de Topografías Médicas, desarrolladas con cierto éxito hasta desaparecer prácticamente en la cuarta década del siglo XX. Con todo, el paradigma de las Topografías Médicas representa una valiosa aportación de estudios empíricos de tipo urbano, y uno de los primeros intentos teóricos de análisis de las interrelaciones entre las personas y el medio en que habitan. El principal fruto del interés por la mejora de las condiciones de vida en las ciudades suscitado por esas Topografías será, sin duda, la elaboración y posterior aprobación en España de la Ley para el Saneamiento, Reforma y Ensanche Interior de 1895.

\section{Los Proyectos de Saneamiento y Reforma Interior}

Aunque en España la plasmación física de esos proyectos en documentos legales no se hará realidad hasta 1895, en Europa existen iniciativas de este tipo desde las primeras décadas del siglo XIX, en primer lugar en Inglaterra, cuna de la revolución industrial, y posteriormente en Francia ${ }^{4}$. Con evidente retraso respecto a la normativa europea, el precedente español de la legislación sobre saneamiento y reforma interior aparece plasmado en el «Proyecto de Ley General de Posada Herrera» de $1861^{5}$. No obstante, el fracaso de esta iniciativa supuso la total fragmentación de los principales cometidos urbanísticos, de forma que la normativa de ensanche pasó a un primer plano, mientras que los temas de reforma interior eran tratados secundariamente. Finalmente, la aprobación en junio de 1879 de la «Ley de Expropiación Forzosa» abriría el camino para la aprobación de la «Ley para el saneamiento, reforma y ensanche interior de las poblaciones» de marzo de $1895^{6}$, con el objetivo principal de «mejorar y sanear las grandes poblaciones en el sentido que demanda la ciencia de la higiene». Como complemento a esta ley se aprobaron otras muchas disposiciones con finalidades diversas ${ }^{7}$, las cuales, junto con las indicaciones contenidas en el «Estatuto Municipal» de $1924^{8}$ y el articulado de las distintas ordenanzas municipales, completarán la normativa sobre saneamiento y reforma interior en las primeras décadas del siglo XX en España.

4 Vid. BASSOLS COMA, M., Génesis y evolución del Derecho Urbanístico Español, 1812-1956, ed. Montecorvo, Madrid, 1976 pp. 360-362.

5 De la lectura del proyecto de Posada Herrera se desprende una evidente conexión entre éste y las iniciativas europeas, al tratar de conjugar en ambos casos saneamiento y construcción de viviendas sociales en un mismo objetivo de reforma de las ciudades. Cf. BASSOLS COMA, M., 1976, ob. cit., p. 363.

6 Cf. Legislación Especial de Ensanche de Poblaciones, Madrid, Centro Editorial de Góngora, 1902, 251 pp.

7 Como fueron, por ejemplo, la legislación sobre «Casas Baratas» de 1911, 1921 y 1924, la Real Orden de agosto de 1923 sobre «Condiciones higiénicas de las viviendas y las condiciones técnico-sanitarias para el ensanche y reforma interior de las poblaciones», la «Instrucción General de Sanidad» de enero de 1904, las «Bases generales para la redacción de los Reglamentos de Higiene» de octubre de 1910, las «trucciones técnico-sanigensy pe eno de 1923, el «eglamento de Sanidad Municipal» de febrero de 1925, o la stey de Bases de la arias» de enero de 1923, el «Reglamento de Sanidad Municipal» de febrero de 1925, o la «Ley de Bases de la Cuya mayor

meyor novedad en relación con la mejora interior radica en la necesidad de formular con antelación un plan general de alineaciones o de reforma interior para emprender cualquier tipo de obras. 


\section{Medidas higiénico-urbanísticas en las principales ciudades alicantinas}

\subsection{El inmobilismo de Orihuela}

Se ha señalado como Orihuela permanece anclada en un régimen agrario y transita por la segunda mitad del siglo pasado sin apenas modificaciones en su callejero. Una clara descripción de la ciudad la ofrece un viajero en 1894:

Poco ruido, mucha reposada calma, nada de chimeneas ni de estruendo de fábricas (...). Orihuela vive en el siglo diez y nueve pero no se halla poseída del vértigo de fin de siglo, vive encantada en la tradición, y su huerta y la pacífica agricultura (...). Atravesando aquella calle algo empinada con sus casucas pequeñas, con aquella confusión de líneas en que para nada se han tenido presente la regularidad ni los preceptos de construcción; dúdase como tal desorden pudiera dar un tan bello conjunto. Sólo falta para completar la ilusión que las mujeres morenas de negros y encendidos ojos, de pelo azulado por la brillantez de su negrura, llevaran trajes más adecuados a la indumentaria arábiga para creeros transportados a una calle de Tetuán o el Cairo, o a los tiempos anteriores a la conquista ${ }^{9}$

La ciudad vive recogida en su callejero tradicional, ceñida por el cauce del Segura a modo de barrera que limita y condiciona su plano y, a la vez, actúa como gran cloaca, función que ha pervivido hasta nuestros días. La atonía demográfica impide, no sólo la realización de un ensanche sino que, incluso, casi paraliza la construcción de manzanas a lo largo de toda la centuria. De hecho, la llegada del ferrocarril en 1884 solo da pie a levantar una alameda y un parque, la Glorieta, en el camino de la estación. Será ya en las primeras décadas del siglo XX cuando la ciudad, de la mano de una cierta prosperidad económica y demográfica, experimente cierto crecimiento urbano y se dote de algunos servicios perentorios desde el punto de vista higienista, como la apertura de un matadero y de una plaza de abastos. Sin plantear reformas sobre el callejero tradicional (que sigue padeciendo hoy día los inconvenientes derivados de un entorno infradotado y en proceso de desmoronamiento poblacional y urbano), la redacción del proyecto de ensanche se demoró hasta $1927^{10}$.

\subsection{El crecimiento fragmentado de Elche}

La ciudad de Elche experimenta a partir de 1877 los beneficios demográficos del incipiente proceso de industrialización. Las reformas urbanas acometen la «regularización» del plano del callejero, intentando acabar con los callejones ciegos, focos de insalubridad y, a la vez, de inseguridad ciudadana: «(...)porque sirve de depósito de inmundicias y de abrigo

9 CASAÑ ALEGRE, J., Recuerdos de viajes por nuestra pátria. Alicante-Orihuela-Murcia, Alicante, 1894, Tip. Antonio Reus, 190 pp., p. 60.

10 DIZ ARDID, E., ALEDO SARABIA, J., Orihuela. Un patrimonio arquitectónico rural y urbano en peligro, Alicante, I.C. Juan Gil-Albert, 1990, 136 pp., p. 25: el proyecto de Ensanche fue elaborado por el arquitecto municipal Severiano Sánchez Ballesta. 


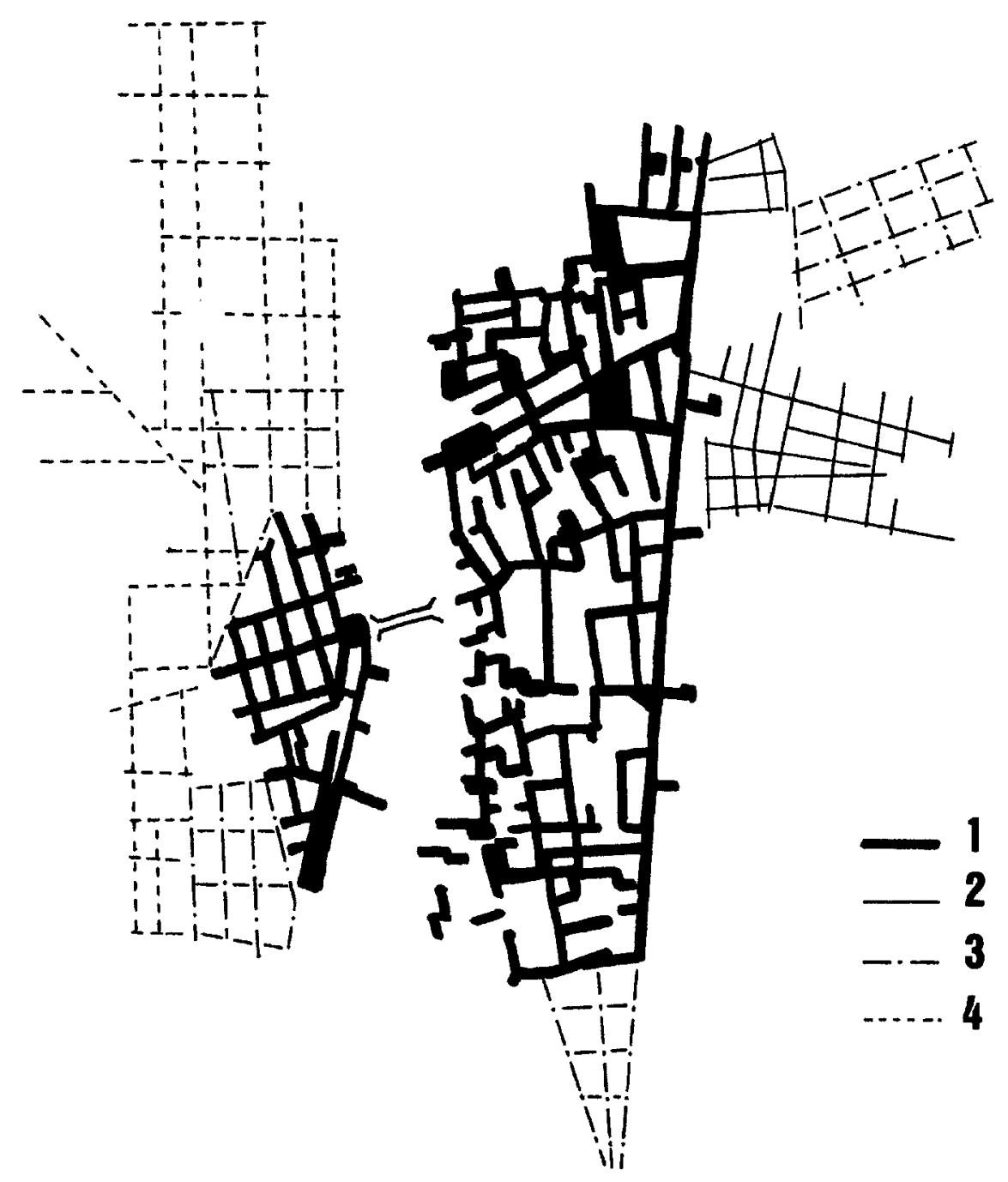

FIGURA 1. El «ensanche fragmentado de Elche»: 1, callejero previo a 1890; 2, calles trazadas en 1890-1905; 3, calles trazadas en 1910-1920; 4, calles trazadas en 1920.1930. Fuente: Gozálvez Pérez, V., ob. cit., 1976; elab. Prop. 
para hombres mal intencionados $(. ..) »^{11}$; de igual manera, se elaboran diferentes proyectos de alineación de calles que, sin embargo, como en tantas otras ciudades, quedaron sin efecto en su mayor parte. En ese sentido, destacada fue la misión desempeñada desde 1855 por la Comisión de Ornato, que se halla en el origen de muchas de las reformas de carácter higienista y estético acometidas en la ciudad, pero también tras las reformas más estrictamente funcionales, como en la alineación de calles y en la mejora de las funciones urbanas $^{12}$. Es en esos años cuando, por el incremento del tránsito rodado, se procura deslindar éste del tránsito peatonal, comenzando una vasta tarea de encintado de aceras y, a la vez, de pavimentación (mediante adoquinado) de las principales calles, como la Corredora, por donde transitaba la carretera Alicante-Murcia. En 1878 se establece en 4,5 m el mínimo de la sección de las calles como referencia para las nuevas y para reformar las existentes, algunas de las cuales se ornan con arbolado.

El crecimiento urbano no se manifiesta en la misma medida que el demográfico, debido al hacinamiento de los inmigrados en el callejero consolidado. No obstante, entre 1890 y 1902 se levantan los pequeños barrios del Carmen, de San Miguel y de Chinchilla a partir de un haz de calles rectilíneas sin llegar a las directrices propias de los ensanches, manifiestas ya en las manzanas construidas a partir del primer decenio del s. XX, origen de los barrios de les Pataes, del Pont Nou, del Asilo, de la Tripa y de la Barrera (ya en 1923), levantados sin embargo como piezas desgajadas, dispersas por los extremos del callejero tradicional, sobre el abanico de caminos en todas direcciones. Se trató de iniciativas concretas y aisladas, debidas a impulsos demográficos puntuales y, con frecuencia, motivados por el interés particular del propietario del suelo: huertos que se urbanizaban sin responder a los criterios de un plan general para toda la ciudad, y que se realizaban según planos cerrados en sí mismos.

La ciudad se había desarrollado a manera de huso de dirección Norte-Sur constreñida por dos directrices fundamentales de igual rumbo: el cauce del Vinalopó por el flanco Oeste, y el trazado de la Acequia Mayor por el Este, tras la que se expanden los huertos del Palmeral.

En 1911 se acuerda elaborar un proyecto de alineaciones y ensanche de la ciudad, especificando una alineación ortogonal de las calles, con una sección mínima de $8 \mathrm{~m}$, que debería extenderse por la derecha del Vinalopó, a partir de los hitos viales del Puente de Santa Teresa y del Pont Nou, que lo comunican con el callejero del centro histórico ${ }^{13}$. No obstante, la insuficiencia del desarrollo normativo de dicho proyecto, así como su escasa puesta en práctica fue responsable de que, en 1922, el Ayuntamiento ilicitano siguiese insistiendo en la necesidad de un plano general para la ciudad.

La llegada de inmigrantes en las primeras décadas del s. XX acentuó la carencia de viviendas y, con ella, los problemas de hacinamiento. En 1908 una acta municipal señala la fuerte ocupación de las viviendas: dos o más familias «en una sola y reducida habitación» $»^{14}$. En esas circunstancias, la iniciativa popular impulsó la constitución en 1913 de la sociedad La Edificadora Obrera Ilicitana, para construir 100 casas de planta baja y patio sobre el Ensanche, aunque dos años después se disolvería la sociedad. Tal iniciativa obrera tuvo, empero, mayor arraigo en las décadas siguientes, y es responsable de la mayor parte

1 GOZÁLVEZ PÉREZ, V., La ciudad de Elche, Valencia, Dpto. de Geografía, 1976, 290 pp., p. 94. Cita referida a las propuestas de 1861 para la demolición del Trinquete.

12 GOZÁLVEZ PÉREZ, V., 1976, ob. cit., p. 91.

PÉREZ, V, 1976, ob.

14 GOZÁLVEZ PÉREZ, V., 1976, ob. cit. p. 116 
del crecimiento urbano de ciudades industriales como Elda, merced a la creación de cooperativas obreras para la construcción de viviendas baratas desde $1916^{15}$.

En otro orden de cosas, las medidas higienistas en la ciudad se manifiestan también por el traslado de cementerios a lugares más alejados, ya que con cierta frecuencia se erigían en focos de contagio, sustituidos por jardines o huertos; y también, como en las demás ciudades, por los intentos de resolver los problemas del abastecimiento de aguas, mediante captaciones de manantiales cada vez más alejados y a través de la ampliación del número de fuentes de uso público.

\subsection{El crecimiento en altura de Alcoy y la insuficiencia de viviendas para los obreros}

Los precedentes teóricos de los intentos de reforma interior en Alcoy son los estudios anexos que se realizaron con objeto de la redacción del Plan de Ensanche de 1878. Este Plan representó el punto de mira exclusivo en materia urbanística de la ciudad durante cerca de cuarenta años, salvo los intentos de reforma interior y las actuaciones puntuales en construcción de casas baratas. De igual modo, el centro de la ciudad había carecido siempre de una mínima atención, y únicamente el Plan de Alineaciones de $1849^{16}$ supuso la mejora relativa en sus condiciones internas, favoreciendo la viabilidad interior por medio del ensanche y alineación de sus principales calles.

Sin embargo, ni las condiciones estructurales y estéticas de los edificios, ni los servicios higiénicos mínimos de las construcciones, ni las dotaciones en espacios libres, zonas verdes y servicios públicos, habían sido mejorados. Por contra, mientras los terrenos del ensanche continuaban vacíos y las nuevas construcciones de viviendas obreras se hacían en número muy reducido, el único punto de acogida de población continuaba siendo el área central de la ciudad, donde las condiciones ambientales se degradaban progresivamente y donde se llegaban a superar densidades de 2.000 hab/Ha. Ilustrativo de este proceso es el hecho de que si bien entre 1888 y 1930 la población crece en unos 8.500 individuos, el número de viviendas lo hace tan sólo en algo más de 500, lo que ofrece una proporción teórica de 17 nuevos moradores por cada nueva construcción entre ambas fechas ${ }^{17}$.

La nueva población llegada a la ciudad, sobre todo a partir de 1910, atraída por la floreciente industria local, incapaz de acceder a una vivienda de nueva planta, debe acomodarse necesariamente en el espacio urbano construido, succión ésta que se realiza de dos modos: la continua subdivisión de las viviendas en estancias para alquilar - las populares cases de clau-y el crecimiento en altura de los edificios, ambas prácticas comunes durantes estas décadas.

Con todo, no será hasta 1927 cuando se lleven a cabo medidas de reforma interior. El Plan de Reforma Interior de Alcoy — «Proyecto de Reforma del Plano de Ensanche y Rectificación del Casco Antiguo de la Ciudad de Alcoy»—de ese año ${ }^{18}$ se asienta en las bases

15 PONCE HERRERO, G., «El Vinalopó Medio», Historia de la Provincia de Alicante, t. 1, Murcia, Ed. Mediterráneo, 1985, pp. 290-296.

16 DAVILA LINARES, J.M. «La ordenación urbanística durante la primera mitad del siglo XX. Premisas para un tratamiento integral de los espacios urbanos», Investigaciones Geográficas, n 9, IUG, Universidad de Alicante, 1991, pp 101-113.

17 DÁVILA LINARES, J.M., Alcoy: desarrollo urbano y planeamiento, Alicante, Universidad de Alicante, 1993, pp. 86-97.

18 Vid. DÁVILA LINARES, J.M., Planeamiento y ordenación urbanística de la ciudad de Alcoy, Tesis Doctoral, 3 vols., Univ. de Alicante, 1990, ff. 578 y ss. 
urbanísticas promulgadas por el Estatuto Municipal de Calvo Sotelo aprobado en 1924, que facultaba a los Ayuntamientos para acometer las obras que fuesen necesarias en materia de reforma interior. Así, además de la «mejora social de la población», se fijó el objetivo de abrir nuevas vías, mejorar las existentes, y la construcción de edificios particulares y de parques y jardines, sirviendo además como futuro plano oficial de alineaciones y rasantes.

El proyecto preveía reformas tanto en el centro de la ciudad como en la zona del Ensanche de 1878. Las indicaciones del plan con respecto al espacio interior pretendían respetar el trazado general de las calles existentes, aunque dotándolas de mayor amplitud y regularidad en sus nuevas alineaciones y rasantes. De otro lado, se dejaba entera libertad para incluir en los proyectos la apertura de nuevas calles en ese espacio construido, «justificando debidamente la necesidad de tal innovación».

El Proyecto de Reforma Interior de Alcoy quedó, no obstante, sin realización práctica, puesto que el recrudecimiento de la crisis económica de finales de los años 1920 en la ciudad y la multiplicación de huelgas y agitaciones sociales, hicieron imposible acometer ninguna obra de carácter urbanístico. De hecho, este receso en la construcción de viviendas y en la urbanización de espacios se prolongará hasta finales de la década de los años 1940, momento a partir del cual comienzan a construise algunos grupos de viviendas sociales, bien de iniciativa estatal o mediante cooperativas.

Con todo, sí se llevaron a cabo operaciones aisladas de reforma interior, no a partir de un planeamiento general previo, pero sí con finalidades concretas. En este sentido puede citarse el ensanche de la calle Santo Tomás, para poner en comunicación el centro urbano con el puente San Jorge - inaugurado en 1931—y, con él, la tercera zona del Ensanche, la cual a raíz de ello verá multiplicar enormemente el número de nuevas construcciones en su seno. Se llevaron a cabo, incluso, regularizaciones del trazado de algunas otras calles y retranqueo de edificios, como en las de San Nicolás, San Lorenzo, San Francisco, etc., pero siempre como operaciones aisladas.

\subsection{Los grandes proyectos de la capital provincial}

El desarrollo urbano de Alicante a comienzos del siglo XIX se hallaba claramente supeditado a intereses militares. Desde la fortaleza de Santa Bárbara descendían dos lienzos de muralla que envolvían el callejero, compartimentándolo en dos distritos: el primero, el casco antiguo, con un caserío prácticamente embutido en las antiguas murallas medievales que, a la altura de la actual Rambla de Méndez Núñez, lo separaban del segundo distrito y barrio más nuevo, el de San Francisco; en general baluartes, acuartelamientos, explanadas, puertas y áreas de servidumbre estratégica condicionaban, junto a la red de caminos preexistentes, el urbanismo civil.

Serán precisamente consideraciones militares las responsables de los importantes cambios urbanos experimentados en Alicante con el inicio de la nueva centuria: la Guerra de la Independencia obliga, por intereses defensivos de la fortaleza y flanco norte de la ciudad, a derribar y dejar expedito el populoso barrio extramuros de San Antón. Al mismo tiempo, se amplía la línea de murallas para cerrar el ángulo formado entre las puertas de San Francisco (en el Camino de Madrid) y de la Reina (en el Camino de Alcoy) y configurar un nuevo trazado del recinto murado, más acorde con la ingeniería militar, en el que dar cobijo a los vecinos desalojados de San Antón. Comenzado a construir en 1811, adoptó un elemental plano ortogonal, trazado a cordel y organizado en torno a la Plaza 

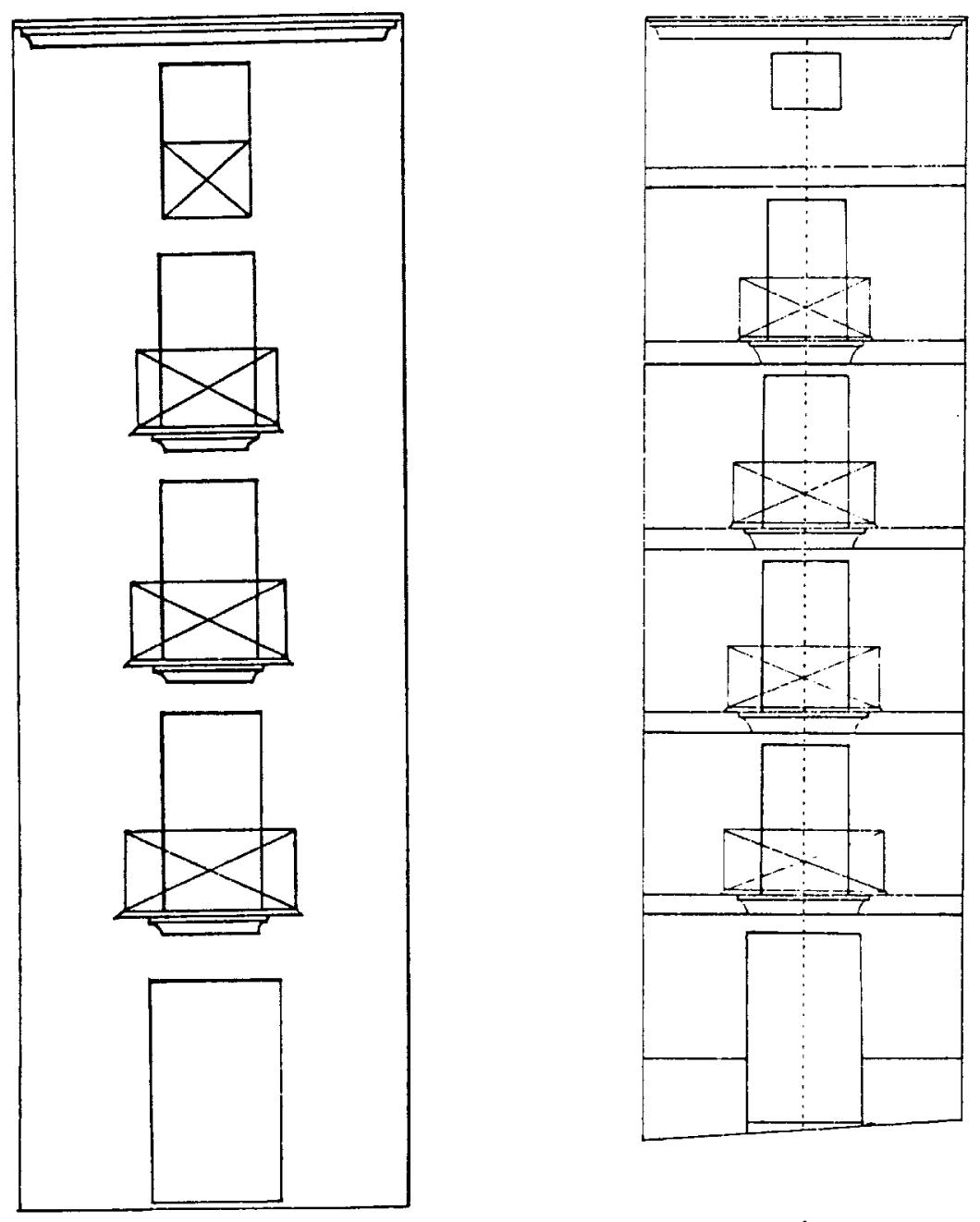

\section{escala de $1 / 0^{\circ}$}

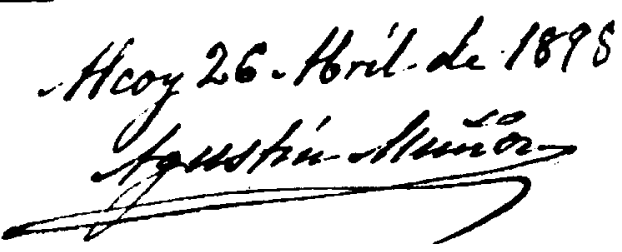

FigURA 2. Edificios de nueva planta típicos en los barrios antiguos de Alcoy, donde se combinan estrechez de fachada (4 ó 5 metros) y gran desarrollo en altura: «tal estrechez, no ha podido menos de originar también una subdivisión ilimitada de los edificios de los particulares, hasta el punto de de originar también una subdivisión ilimitada de los edificios de los particulares, hasta el punto de
que en gran parte de ellos, el número de sus propietarios, se cuenta por el número de sus pisos ó tal vez de sus habitaciones ó departamentos...» (Archivo Municipal de Alcoy, Ordenanzas 1819-1889, inf. 12-IV-1880). 
Nueva, con importantes problemas de conexión con el entramado urbano preexistente ${ }^{19}$, si bien se adoptan ya en él importantes soluciones urbanísticas, como son su trazado de calles largas, rectas y de mayor amplitud, acordes con las nuevas tendencias del diseño urbano.

La pérdida de valor militar como plaza fuerte relegó a la ciudad a un prolongado olvido por parte del Gobierno de España que, sumado a la carencia de iniciativa de los políticos locales, de la que se queja insistentemente el que sería arquitecto municipal, Guardiola Picó, impidieron a Alicante la asunción plena de las reformas urbanas propugnadas en ese siglo por el Higienismo y por el crecimiento demográfico, medidas precisas exigidas, según Guardiola Picó, «por la necesidad, la higiene, el ornato y por último la justicia $\gg^{20}$.

La preocupación del citado arquitecto por la ciudad es manifiesta a lo largo de las dos últimas décadas de la centuria y primera del siglo XX, expresada en numerosos proyectos de reformas internas y dotación de servicios, que van desde su participación en el concurso de Planes de Ensanches de 1887, hasta la publicación de diversos artículos en los diarios El Liberal y El Imparcial sobre aspectos urbanísticos puntuales, y varias publicaciones posteriores de mayor entidad. Junto a él, y a veces en colaboracion, en las últimas décadas de la centuria anterior se alzan otras voces que evidencian las precarias condiciones higiénicosanitarias de la ciudad, entre las que destacan, E. Manero y su Estudios sobre la topografía médica de Alicante (1883), P. Carreras y su Medios para mejorar las condiciones higiénicas de Alicante (1886), E. Sánchez Santana, que publicó junto a Guardiola Picó la Memoria higiénica de Alicante (1894), etc. La preocupación se extiende a la imperiosa necesidad de agua para el abasto de Alicante. En este sentido, destacados fueron los estudios de Pardo y Gimeno en 1880, Proyecto de traída de aguas de la Alcoraya a Alicante y los de Lastres y Juíz en 1894, Abastecimiento de aguas para la ciudad de Alicante. Sin olvidar otra serie de trabajos orientados a la difusión de las excelencias climáticas de Alicante y su aplicación práctica a la salud, como exponen Sañudo Autrán en su Alicante, estación invernal, Sánchez Santana, en su Residencia invernal de Alicante y el médico Federico Parreño en 1889: "Alicante tiene en su atmósfera un tesoro que explotar» ${ }^{21}$, en línea con otros autores y con la «comisión municipal» nombrada al efecto, que intentaban impulsar la función lúdico-recreativa asociada a la climatoterapia y a la balneoterapia predecesora del actual fenómeno turístico.

De ese conjunto de obras y, especialmente, de los trabajos de Guardiola ${ }^{22}$, puede sintetizarse la situación higiénico-sanitaria de la ciudad, los grandes problemas que la aquejaban e incluso, en ocasiones, los proyectos y reformas propuestos para darles solución. En este sentido, los planes concretos y las previsiones pueden agruparse en tres conjuntos, atendiendo al ámbito espacial afectado.

19 CALDUCH CERVERA, J.; VARELA BOTELA, S., Guía de Arquitectura de Alacant, Alicante, C.S.I., 1979, t. I, p. 77

20 GUARDIOLA PICÓ, J., Alicante en el siglo venidero, Alicante, Imp. Galdó Chápuli, 1897, pp. 288 y ss

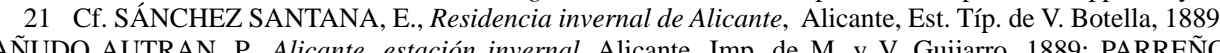
SANUDO AUTRAN, P., Alicante, estación invernal, Alicante, Imp. de M. y V. Guijarro, 1889; PARRENO .

22 Véase, además de los citados, GUARDIOLA PICO, J. (1895), Cuestionario propuesto por el Excmo. Sr. Ministro de la Gobernación..., Alicante, Establ. Tip. Costa y Mira, 1895, 46 pp.; y Reformas en Alicante para el siglo XX, Alicante, Imp. J.J. Carratalá, 1895, 88 pp. 


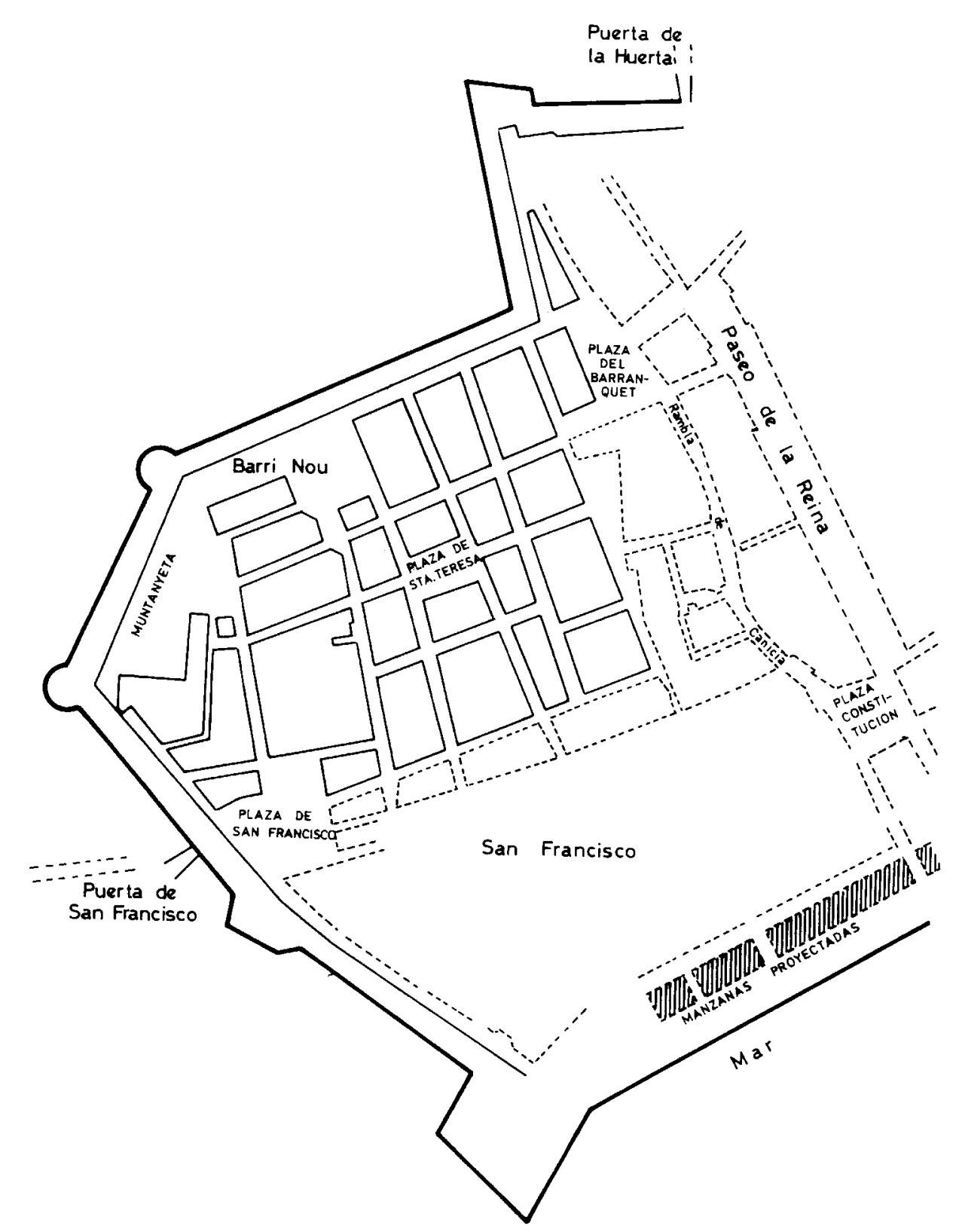

FIGURA 3. Alicante a mediados del s. XIX. En línea discontinua el sector urbano afectado por las reformas internas precisas para una adecuada conexión entre el centro histórico y el Barri Nou. Elab. Prop. 


\subsubsection{Reformas de carácter general}

Entre los problemas urbanísticos de índole general, con repercusión en los ámbitos de la salud, destaca la carencia en el suministro de aguas potables. El abasto de agua para el consumo urbano pudo satisfacerse en la primera mitad de siglo con los manantiales ubicados en el propio término municipal y aún en los confines de la ciudad. No obstante, en el año 1867 se consumó el agotamiento de la mayor parte de esas fuentes ${ }^{23}$, quedando relegado el suministro a $558 \mathrm{~m}^{3} /$ día, procedentes de la Mina del Sagrat y de La Alcoraya, para un total de 49.463 habitantes ${ }^{24}$, lo que supone una relación de 11,3 1/habitante y día, cuando en la actualidad el consumo medio estimado en Alicante oscila entre los 250 y 300 1/hab/día ${ }^{25}$. Tal limitación afectaba a la dinámica urbana, condicionando el crecimiento de la ciudad y del Ensanche planificado, al fomento de la industria y los servicios y, de manera significativa, a la propia higiene y salud comunitaria, como señala Guardiola: «Desgraciadamente, la ciudad de Alicante carece de agua, no ya para beber, sino para los demás servicios $»^{26}$; y cuando comenta los efectos en la ciudad de la traída de aguas: «...transformará su modo de ser y será base de su higiene» ${ }^{27}$. Tal cuestión afectaba, asimismo, a la limpieza y policía de las calles y, de manera más grave para la salud, por la insuficiencia de fluido para arrastrar los residuos de las alcantarillas. La solución llegaría en 1898 con la apertura del acueducto que llevará a la ciudad las «Aguas de Sax», primer paso de futuras captaciones más alejadas.

Como se ha indicado, el alcantarillado constituía también notable inconveniente para la salud: «Si la función de un alcantarillado es conducir lejos todos aquellos productos que puede saturar la atmósfera de miasmas morbosos (...) las alcantarillas de Alicante no cumplen ni pueden llenar ese objeto, siendo por el contrario una amenaza constante para la salud, y un foco perjudicial, no sólo para los habitantes de la población, sino también para las tripulaciones de los barcos... $\gg^{28}$. Tal aseveración del arquitecto municipal estaba sustentada en el caótico trazado de la red de cloacas, en la escasa pendiente de éstas en la zona baja de la población, en su insuficiencia para recoger los depósitos de todo el casco urbano que, en su mayor parte, seguía utilizando pozos negros, en la señalada carencia de agua para facilitar los arrastres y en el desagüe de casi todos los colectores en la dársena del puerto, quedando muy limitada la disolución de las materias fecales ante la inexistencia de corrientes marinas que renovasen las aguas. Así, señala que «(...) nuestras alcantarillas resultan ser pozos negros de grandes dimensiones, que abarcan la longitud de las calles (...) es preciso tener perfectamente cerrados los tragaderos de las calles a menos de vivir en una atmósfera de repugnante y anti-higiénica podredumbre»²9. Pero a la vez, el vertido de esas aguas fecales a la dársena del puerto, convertía esa lámina de agua en otro foco de insalubridad, precisamente en la fachada burguesa de la ciudad. En ese sentido el Proyecto de Obras para el saneamiento del Puerto de Alicante y de su zona marítima elaborado en 1905 por Ramón Montagut expone: «No puede ni debe consentirse que un puerto de la importancia de éste no pueda recorrerse en los días calurosos del verano, y aun en algu-

23 GUARDIOLA PICÓ, J., 1895, ob. cit., pp. 11-15.

24 Según el Censo de 1897, I.N.E.

25 JUÁREZ SÁNCHEZ-RUBIO, C. y otros, El reto del agua. Alicante, Inst. Juan Gil-Albert, 1989, p. 83.

26 GUARDIOLA PICÓ, J., 1895, ob. cit., p. 11.

27 GUARDIOLA PICÓ, J., 1897, ob. cit., p. XII.

28 GUARDIOLA PICÓ, J., 1895 , ob cit., p. 17.

29 GUARDIOLA PICÓ, J., 1895, ob. cit., p. 17. 


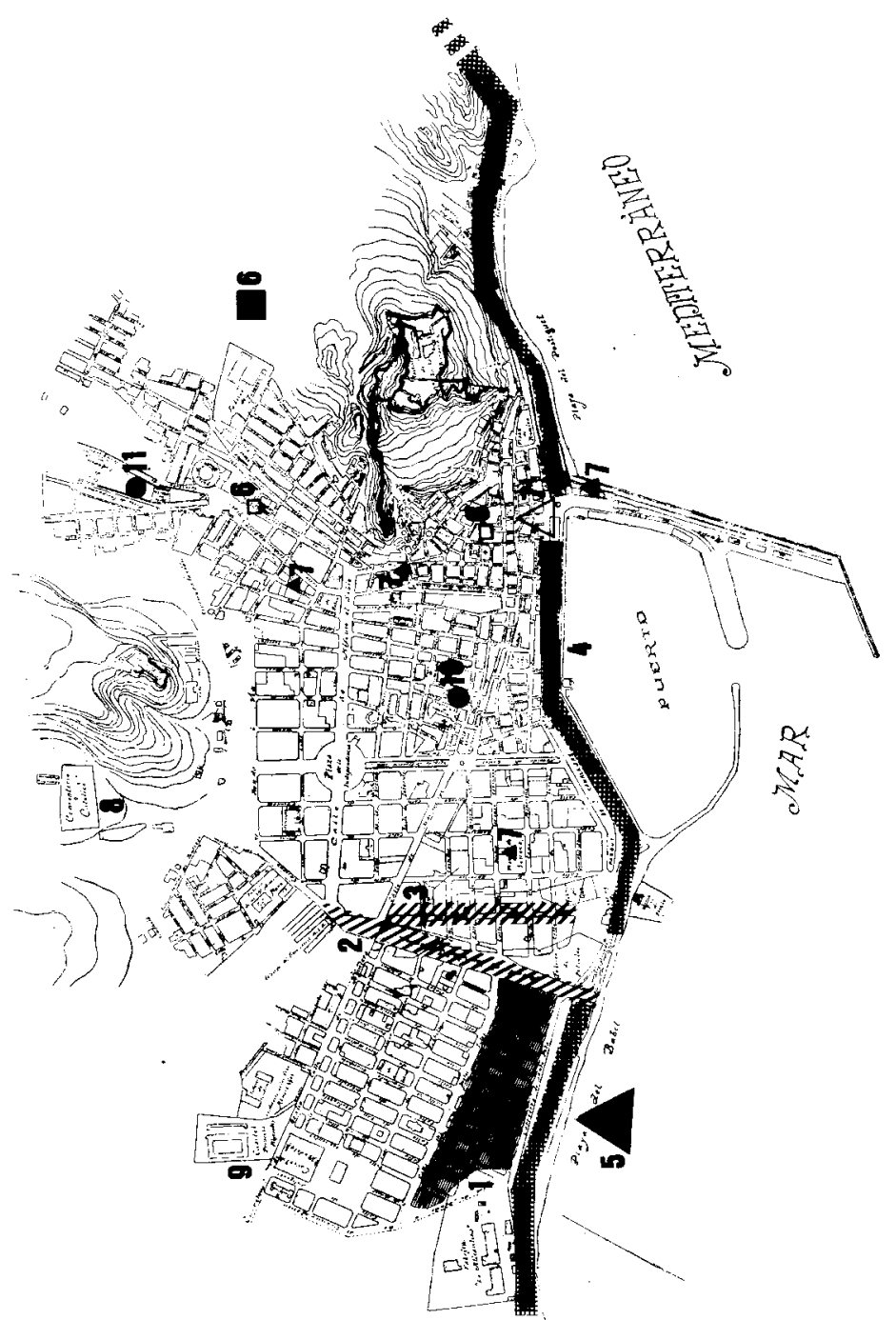

FigURA 4. Los grandes proyectos de reforma urbana para Alicante en 1895: 1, Parque en el Camino de Santa Pola; 2, apertura de la Avda. Marqués de Salamanca y entubado de la rambla; 3, ampliación de la C/. Churruca al servir de conexión entre las estaciones del ferrocarril y de autobuses; 4 , tratamiento de toda la fachada marítima, con la construcción de un paseo «extenso, higiénico y cómodo» de $5 \mathrm{~km}$ de longitud, extendido por los caminos de Santa Pola y Valencia; 5, instalación de un «Sanitarium» en la Playa de Babel, para potenciar la ciudad como estación balnearia: 6, traslado de los hospitales Provincial y Militar desde San Nicolás y San Antón hasta el Pla del Bon Repòs; 7 , traslado del Mercado de abastos desde la Puerta del Mar y creación de cuatro mercados: en San Antón, en la Plaza de Séneca, en el centro histórico y en el Muelle de Levante; 8, construcción de un nuevo cementerio, y clausura de el de San Blas; 9, construcción de un nuevo Cuartel de Militares y cambio de uso de los edificios militares del momento, dedicados a escuelas y jardines: 10, instalación del Parque de Bomberos; 11, Casa de Beneficencia y Hospício. Elab. Prop. 
nos del invierno, según el estado de la atmósfera y dirección del viento, sin sufrir el intenso, nauseabundo y repugnante hedor de las alcantarillas que enturbian las aguas y las ennegrecen... $»^{30}$. El autor cuestiona seriamente la fama de Alicante como ciudad sana que, en su opinión, debe al hecho exclusivo de ser destino invernal de personas aquejadas de enfermedades de las vías respiratorias. En su proyecto propone una mejora integral de la red de alcantarillado y la derivación de sus vertidos hacia el exterior de la ciudad (paraje de l'Aigua Amarga).

Cuestión de agravio general era asimismo el estado de policía de las calles. Sólo las más céntricas estaban pavimentadas y en todas la suciedad constituía rasgo común. Guardiola aboga por la pavimentación general, y propone diferentes sistemas, así como por la regulación del tránsito rodado y la creación de unas brigadas municipales que garantizasen el aseo de la ciudad: «La falta de limpieza es otro mal que lamentamos, siendo como es, primer e indispensable elemento parta la higiene y bienestar» ${ }^{31}$.

\subsubsection{Reformas sectoriales}

Especialmente destacados, desde el punto de vista higienista, fueron los proyectos urbanísticos del Ensanche y barrios de Benalúa, Pla del Bon Repós, Ciudad Jardín, y la propia remodelación del casco antiguo. Para el primero, esencial fue la demolición de las murallas de la ciudad, autorizada en 1858, que permitía la expansión orgánica de la nueva «ciudad abierta». Aprobado en 1898, el proyecto de Ensanche alicantino se incorporó tarde a la ciudad y, aún, con escasos resultados, como señalan Calduch y Varela, por su reducido tamaño, escasez de dotaciones públicas y mala tramitación, de forma que, contrariamente a lo previsto, acabó ocupado por insalubres establecimientos fabriles y de almacén ${ }^{32}$.

Mejor fortuna tuvo el Barrio de Benalúa, comenzado a edificar en 1884, que asume las características generales de los ensanches y del higienismo. Cuenta con una retícula viaria ortogonal, de calles amplias y aceras arboladas, con muy bajas densidades y viviendas sobredimensionadas $\left(175 \mathrm{~m}^{2}\right)$ en comparación con las de la ciudad consolidada en la época. El barrio, ubicado en un altiplano bien aireado, separado de la ciudad y próximo al mar, ha sido considerado como la primera promoción de «casas baratas» en España ${ }^{33}$.

Ligados a esos modelos urbanísticos se encuentra el plan de reordenación del caserío y terrenos del Pla del Bon Repós, de Vicente Santafé en 1912, y la creación de la Ciudad Jardín en 1920 por Fajardo Guardiola ${ }^{34}$, manifestaciones algo más tardías de las doctrinas higienistas y de las ideas de la «ciudad-jardín». En todos los casos son proyectos nuevos que se ubican de manera sintomática lejos del casco urbano, en busca de suelo barato y apto, así como para potenciar la idea de ciudad nueva.

Frente a esas soluciones, el casco antiguo padecía todavía todos los inconvenientes derivados de las altas densidades - rayanas en el hacinamiento en algunos distritos-, de la

30 MONTAGUT, R., Proyecto de obras para el saneamiento del Puerto de Alicante y de su zona marítima, Doc. 1, «Memoria», p. 2, en NAVARRO VERA, J.R., El saneamiento de Alicante de 1905: ciencia, técnica e higienismo, Alicante, C.I.C.C.y P., 1991, 120 pp.

31 GUARDIOLA PICÓ, J., 1897, ob cit, p. 6

32 CALDUCH CERVERA, J., VARELA BOTELLA, S., 1979, ob. cit., t. I, pp. 61-66.

33 CALDUCH CERVERA, J., VARELA BOTELLA, S., 1979, ob. cit., t. I, p. 54. No obstante, en Alcoy se edificaron algunas de estas «casas baratas» ya en 1871, no como promoción cerrada, pero sí como actuaciones puntuales, si bien con idéntica filosofía. Cf. DÁ VILA LINARES, J.M. 1990, ob. cit., vid. pp. 310 y ss.

34 CALDUCH CERVERA, J., VARELA BOTELLA, S., 1979, ob. cit., t. I, pp. 79-80. 
estrechez de las calles, de la pequeñez de las viviendas, de las carencias de las redes de agua potable y alcantarillado, como indica Guardiola, al proponer el derribo de algunas manzanas entre la Plaza de San Cristóbal y la Rambla: «la Higiene ganaría, porque esas calles son sombrías y estrechas y las viviendas reducidas y con escasa luz» ${ }^{35}$. A ello debe sumarse el riesgo de inundación por arroyada de algunas calles y la persistente humedad en toda la parte baja de la ciudad. No obstante, ante las dificultades para acometer reformas de envergadura, las actuaciones quedaban constreñidas a sencillas alineaciones de rasantes y ensanchamientos de algunas calles ${ }^{36}$

Especialmente degradados se hallaban los bordes de la ciudad, incluida la fachada marítima, tanto por las actividades en ella instaladas - fabriles, refinamiento de crudos, almacenamiento y las propias del puerto-, como por falta de aseo general, como recoge Guardiola en sus escritos: «la vía del puerto obligó a dar otra forma al antiguo Malecón, depósito entonces de toda clase de inmundicias (...) y (...) la calle del Socorro cuyo desmonte sólo sirve de depósito de basuras y otros usos que la decencia impide decir más claro (...)» $\gg^{37}$. A ello debe sumarse el desagüe en precario de los diversos colectores de la red de alcantarillado que, descendiendo de las laderas de los cerros interiores, abocaban directamente «en la playa del Postiguet, el puerto y la playa de Babel, en las cuales es constante el movimiento de gentes y tráfico» ${ }^{38}$.

Así, Guardiola, por evidentes motivos higiénicos y también guiado por el afán de promocionar el atractivo de la ciudad como estación balnearia, propuso en diferentes ocasiones la construcción de un extenso parque lineal, con continuidad desde la Playa de Babe —donde el médico Sánchez Santana había diseñado su ciudad sanitarium - hasta la Albufereta, recuperando la ciudad la propiedad del Paseo de los Mártires (en manos de las autoridades de Marina y Fomento), plantando «pinos y palmeras» en la Explanada y prolongando el Paseo de Gomis «hasta más allá del Matadero», con continuidad por el proyectado camino de la Albufereta al pie de la Sierra de San Julián. El parque sería de mayor anchura entre el Camino de Santa Pola y el borde del Barrio de Benalúa, entre la fábrica La Alicantina y la Avenida de Salamanca, para crear un « (...) sitio ameno, pintoresco, e higiénico sobre todo» y conseguir con todo el conjunto una «explanada de grandísima importancia, y de condiciones higiénicas tan superiores, que difícilmente se habría de encontrar otra igual (...) pudiendo así los inmigrantes de una y otra estación hallar bienestar envidiable y disfrutar de un panorama tan encantador y magnífico (...) $x^{39}$.

\subsubsection{Reformas de carácter puntual}

Otras reformas precisas por la precariedad, y aún la ruina, son señaladas para edificios civiles, militares y sanitarios. Las carencias higiénicas en los centros de salud eran alarmantes y gravísimas ya a ojos de los contemporáneos. Entre otras críticas, como muestra, Guardiola señala respecto al Hospital Civil «que los retretes son de sistema ya desechado e insoportables los olores que se perciben y que algunos de dichos retretes están dentro de las salas, se vendrá en conocimiento de lo peligroso que es este Hospi-

35 GUARDIOLA PICÓ, J., 1897, ob. cit., p. 227.

36 RAMOS HIDALGÓ, A., Evolución urbana de Alicante, Alicante, I. Juan Gil-Albert, 1984, pp. 198-210.

37 GUARDIOLA PICÓ, J., 1895 , ob. cit., p. IX y p. 1.

38 GUARDIOLA PICÓ J. 1895 , ob cit., p. 15

39 GUARDIOLA PICÓ, J., 1897, ob. cit., pp. 70-72 y pp. 100-105. 
tal (...)»; y de la Casa de Beneficiencia comenta irónico que «(...)con sólo decir de éste último, que no se comprende como no mueren por el contagio o por la infección los desgraciados seres que a él acuden, está todo dicho» ${ }^{40}$. Partícipes de condiciones parecidas se hallaban el Hospital Militar, la Casa de Socorro, los cuarteles militares, la Cárcel, el Cementerio (en San Blas), el Mercado, las escuelas y otros servicios públicos. Para todos ellos da soluciones, que pasan las más de las veces por construir nuevos edificios y recintos, dotados de los adelantos del momento y siempre guiados por el precepto máximo de la higiene.

Guardiola considera el olvido estatal y la decadencia económica de la ciudad y su puerto responsables principales de las carencias urbanas, pero también resalta el conformismo y la falta de iniciativa de las autoridades locales, que privaron a la ciudad durante décadas, e incluso de forma definitiva, de las reformas que, en su opinión, «la necesidad, la higiene, el ornato y por último la justicia exigen y reclaman de continuo» ${ }^{41}$. Pese a lo expuesto, las exportaciones marítimas alcanzaron un espectacular incremento entre 1854 y 1892, gracias al tratado comercial para la exportación de vinos a Francia. El auge mercantil fue seguido de constantes reformas y ampliaciones en las instalaciones portuarias y, en 1880, el puerto de Alicante fue incluido entre los de «interés general de primer orden $»^{42}$. De ello se deduce que los intereses comerciales e industriales asentados en la fachada marítima pesaron más que los estéticos e higienistas impulsados por los proyectos de reforma urbana.

$$
* * *
$$

En definitiva, puede concluirse que pese a la existencia de una clara conciencia sobre las necesidades de paliar unas lamentables condiciones higiénicas, las reformas en cada uno de los núcleos urbanos analizados fueron siempre a remolque de pequeños intereses económicos, tal como acontecía en otras ciudades españolas. La conciencia crítica aparece en urbanistas y pensadores, no obstante, la inercia en los políticos o la incapacidad de algunas sociedades de vislumbrar la configuración de una ciudad moderna, a partir de las estructuras pueblerinas en que viven inmersas, hizo que la respuesta a esas carencias acuciantes se viese siempre demorada o mal satisfecha, desaprovechando la oportunidad de transformar la ciudad.

En el tránsito del siglo XIX al XX la carencia de viviendas y el hacinamiento son los rasgos más característicos de las sociedades urbanas analizadas, con un corolario de afecciones sanitarias. Tal circunstancia pudo ser paliada mediante planes de urbanismo fundamentados en aspectos higienistas, tal como propugnaban las directrices legales existentes. Por otro lado, la construcción de viviendas baratas pudo ser entendida también como un próspero negocio por parte de la burguesía, como de hecho ocurría en las grandes ciudades españolas. Sin embargo, en Alicante, Elche o Alcoy tales iniciativas fueron siempre reducidas, muchas de ellas llevadas de la mano de los propios obreros agrupados en cooperativas.

40 GUARDIOLA PICÓ, J., 1897, ob. cit., pp. 23 y 24.

41 GUARDIOLA PICÓ, J., 1897, ob. cit., p. 288.

42 PONCE HERRERO, G., «El sector terciario, motor del desarrollo», Comarca l'Alacantí, Alicante, Mancomunidad, 1990, p. 154 


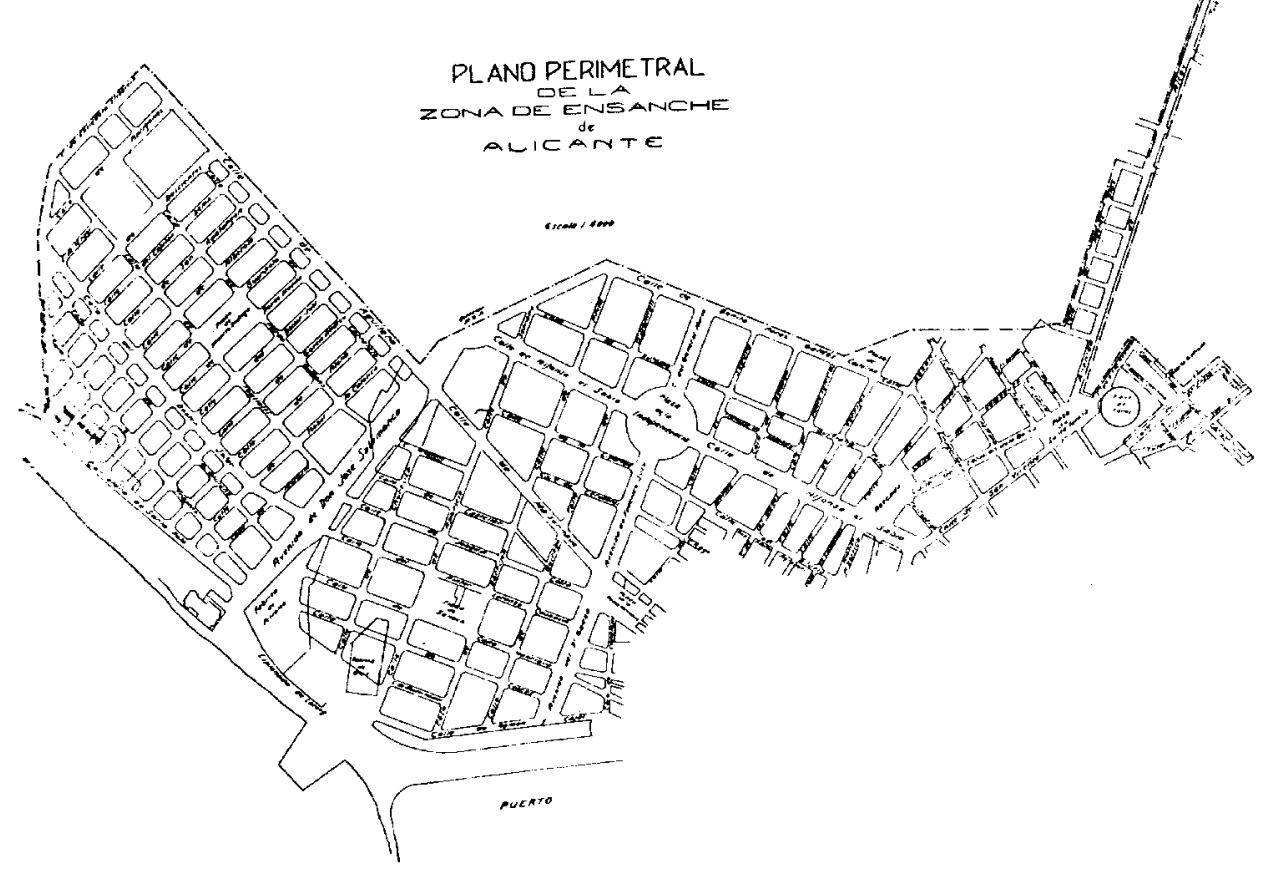

Figura 5. Trazado definitivo del Ensanche de Alicante, definido en 1928, en el que se ajustan los dos anteproyectos elaborados en 1899 por González Altés por Guardiola Picó. Fuente: A.M.A.

Así, los centros históricos se vieron desbordados por las fuertes densidades humanas, al constituir casi el único recurso de la clase obrera para encontrar morada, si bien en condiciones de extrema precariedad y elevado hacinamiento. Esas circunstancias, patentes en cada una de las ciudades que experimentan el tránsito hacia urbes industriales (o con funciones industrial y comercial como Alicante), mantuvieron vivas las teorías higienistas, y en el tránsito de siglo, los proyectos de reforma interior se convertirán por tanto en valedores de una nueva concepción del espacio urbano, en manifiesta simbiosis con unas normas mínimas de habitabilidad y disfrute público, bien que siempre por delante de las concrecciones de esas ideas en los espacios urbanos. De hecho, la lentitud y parquedad de los logros en esa materia permiten concluir que los criterios higienistas, por sí solos, no fueron suficientes para acometer las necesarias reformas urbanas. Por el contrario, éstas llegaron cuando la renovación de la escena urbana se percibió como una posibilidad más de obtener beneficios en la lógica del capitalismo burgues. 